\title{
In vivo imaging of cell morphology and cellular processes in Caenorhabditis elegans, using non-linear phenomena
}

\author{
G. Filippidis ${ }^{\text {a,* }}$, E.J. Gualda ${ }^{\text {a }}$, M. Mari ${ }^{\text {a,c }}$, K. Troulinaki ${ }^{\text {b }}$, C. Fotakis ${ }^{\text {a,c }}$, N. Tavernarakis ${ }^{\text {b }}$ \\ ${ }^{a}$ Institute of Electronic Structure and Laser, Foundation of Research and Technology-Hellas, P.O. Box 1385, 71110 Heraklion, Crete, Greece \\ ${ }^{\mathrm{b}}$ Institute of Molecular Biology and Biotechnology, Foundation of Research and Technology, 71110 Heraklion, Crete, Greece \\ ${ }^{\mathrm{c}}$ Department of Physics, University of Crete, Greece
}

\section{A R T I C L E I N F O}

Article history:

Received 8 April 2009

Received in revised form 3 June 2009

Accepted 10 June 2009

\section{Keywords:}

THG

SHG

TPEF

Imaging

Caenorhabditis elegans

Neurodegeneration

\begin{abstract}
A B S T R A C T
We present the detailed imaging of structures and processes of the nematode Caenorhabditis elegans (C. elegans) using non-linear microscopy. Complementary information about the anatomy of the nematode was collected by implementing a combination of two photon excitation fluorescence (TPEF), second and third harmonic generation (SHG and THG) image contrast modes on the same microscope. Three-dimensional (3D) reconstructions of TPEF, SHG and THG images were also performed. Moreover THG imaging technique has been tested as a potential, novel, non-destructive diagnostic tool for monitoring cellular processes in vivo, such as neuronal degeneration.
\end{abstract}

(c) 2009 Elsevier Ltd. All rights reserved.

\section{Introduction}

The use of femtosecond (fs) lasers for excitation has not only improved the resolution and 3D imaging capabilities of microscopy by multiphoton excitation - e.g., two- or three photon excitation fluorescence (TPEF-3PEF) - but has also demonstrated the possibilities for new detection techniques by exploiting non-linear excitation effects, e.g., second harmonic generation (SHG) and third harmonic generation (THG). The non-linear imaging techniques represent the forefront of research in cell biology. These nondestructive modalities comprise a powerful tool for elucidating structural and anatomical changes of biological samples and for probing functions and developmental processes in vivo at the microscopic level. Consequently, the investigation of in vivo cellular and sub-cellular activities, by means of these non-linear imaging techniques, can provide valuable and unique information related to fundamental biological problems, leading to the development of innovative methodologies for the early diagnosis and treatment of several diseases.

The basic principle underlying these techniques is that for tightly focused fs laser pulses, the photon density is high enough to induce multiphoton absorption or other non-linear (coherent) processes within the focal volume. Fluorophores whose excita-

\footnotetext{
* Corresponding author. Tel.: +30 2810391320; fax: +30 2810391305 . E-mail address: filip@iesl.forth.gr (G. Filippidis).
}

tion maximum is in the ultraviolet (UV) or in the visible (VIS) can be excited by two or three infrared (IR) photons. Since nonlinear absorption and thus induced fluorescence occurs solely at the focal volume of the laser beam, a high axial resolution and consequently the 3D imaging capability of confocal microscopy can be attained without the use of a confocal aperture. Furthermore, there is no interfering fluorescence from the surrounding structures and "out of focal plane" photobleaching/ toxicity can be significantly reduced. More precisely, for nonlinear techniques, the efficiency of the generated signal scales non-linearly with the intensity of the excitation beam. Thus, the use of fs lasers enables high peak powers for efficient non-linear excitation, but at low enough energy that biological specimens are not damaged. Additionally, the use of IR light permits a high penetration depth into tissues due to the low absorption by the primary cellular components (water, proteins, etc.). For SHG and THG, an additional advantage derives from the fact that no energy is deposited (absorbed) by specimens, thus sample disturbance (e.g., thermal, mechanical side-effects) are minimal. Non-linear (TPEF, SHG, and THG) imaging techniques have been used as tools for the in vivo imaging and mapping of sub-cellular biological structures and processes (Campagnola and Loew, 2003; Debarre et al., 2005; Gualda et al., 2008b; Mohler et al., 2003; Psilodimitrakopoulos et al., 2009; Reshak et al., 2009; Sun et al., 2004; Zipfel et al., 2003) providing very promising results.

In our previous works (Gualda et al., 2008a, 2007) non-linear image contrast modalities have been used for imaging of $C$. elegans 
structures and processes by employing mostly a $x y z$ motorized stage (limited resolution to $1 \mu \mathrm{m}$ ). In the current study, that represents a continuation of our previous research, highresolution mapping of the nematode $C$. elegans, in both its anterior and posterior body parts, has been performed. We realized measurements to the nematode by employing a galvano-mirror scanning system in order to improve the quality, the time of data acquisition and the spatial resolution of the recorded non-linear images. Unique, complementary, more accurate information related to the structure and the functions of tissues and specific cell types of the nematode were extracted, by combining three different image contrast modes (TPEF, SHG, and THG) in a single instrument. Additionally, through the development of our experimental apparatus, it was feasible the creation of three-dimensional (3D) reconstructions of combined TPEF, SHG and THG images from the worm. These 3D reconstructions provided valuable, detailed, consummative information for the anatomy of the nematode.

Moreover, THG imaging modality has been tested as potential diagnostic tool for the in vivo, non-destructive, precise identification of the degenerating neurons of the nematode. In a previous work (Gualda et al., 2008b) monitoring, in real time, of the progression of degeneration in C. elegans larvae, by employing THG imaging modality, was showed. Here we present the in vivo 3D reconstruction of a degenerative neuron in the posterior part of the nematode via THG measurements.

\section{Materials and methods}

\subsection{Experimental apparatus}

The experimental set-up (Fig. 1) consists from an Amplitude Systems femtosecond laser operating at $1028 \mathrm{~nm}$. This source is a compact diode-pumped femtosecond laser oscillator delivering a train of high peak power, short duration pulses. The average power of the laser is $1 \mathrm{~W}$, with pulse duration of less than $200 \mathrm{fs}$ and a repetition rate of $50 \mathrm{MHz}$. The femtosecond laser system, emitting at $1028 \mathrm{~nm}$, employed for the realization of the non-linear measurements due to the advantage that its third harmonic signal is in the near UV part of the spectrum $(\sim 343 \mathrm{~nm})$. Consequently, there is no need to use UV optics with special coatings for the collection of the THG signal. Moreover, by employing this excitation wavelength $(1028 \mathrm{~nm})$ the absorption of the water, which leads to unwanted thermal heating of the biological samples, is constrained.

The beam is directed to a modified optical microscope (Nikon Eclipse ME600D) using suitable dichroic mirrors, and is focused tightly onto the sample by an objective lens with high numerical aperture (Nikon $50 \times$ N.A. 0.8 ). To ensure that the back aperture of

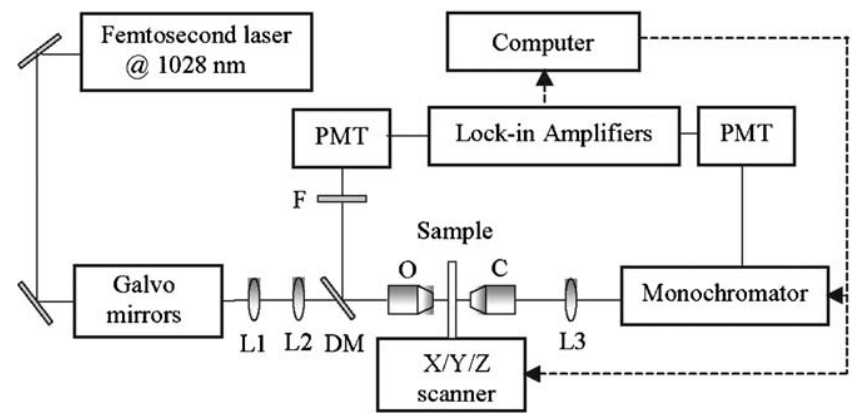

Fig. 1. Schematic representation of the experimental set-up. PMT: photomultiplier tube, DM: dichroic mirror, O: objective, C: condenser, F: filter and L1/L2: telescope lenses, and L3: lens. the objective is fulfilled a telescope system has been used. The average laser power on the specimen is $30 \mathrm{~mW}(0.6 \mathrm{~nJ}$ per pulse). No damage in the sample is observed at this power.

A CCD camera (PixeLINK PL A662) is used for observation. Biological samples are placed between two round glass slides (Marienfeld $0.06-0.08 \mathrm{~mm}$ ) that fit into a translation stage by employing a special holder. In order to get high-resolution images, the scanning is performed by a pair of galvanometric mirrors (Cambridge Tech. $6210 \mathrm{H}$ ), computer-controlled by specially designed software (National Instruments, Labview 7.1). Alternatively, lower resolution images can be recorded moving directly the sample with the $x y z$ motorized translation stage (Standa 8MT167-100). This configuration limits the resolution to $1 \mu \mathrm{m}$, but allows scanning bigger areas, without aberration due to the edges of the aperture of the objective lens.

TPEF signals are collected in the backward direction using a photomultiplier tube (PMT Hamamatsu R4220) connected to a Lock in Amplifier (SR810 Stanford Research Systems). The use of a Lock in Amplifier provides a very good noise rejection, and measurements can be performed in less stringent lighting conditions. The photomultiplier tube is attached at the position of the microscope eye-piece. A short pass filter (SPF $650 \mathrm{~nm} \mathrm{CVI)} \mathrm{is}$ placed at the photomultiplier input to cut off the reflected laser light. Furthermore, a long pass filter (LP $520 \mathrm{~nm}$ CVI) is used for the elimination of reflected SHG and THG signals. For thin samples, most of SHG and THG signals propagate with the laser and are collected and collimated by employing a common condenser lens (Carl Zeiss Plan-Apochromat $100 \times$ N.A. 1.4 oil immersion). A dichroic mirror (Thorlabs) is used to reflect the transmitted beam. The signals are focused by means of a lens (UV Fused Silica PlanoConvex, Thorlabs) into the slit ( $100 \mu \mathrm{m})$ of a monochromator. The focal length of the lens is $3 \mathrm{~cm}$. The monochromator (Digikrom CM110 CVI) consists of two gratings (1200 grooves/mm each). The first one is suitable for visible light (maximum efficiency $80 \%$ at $500 \mathrm{~nm}$ ) and the second is appropriate for ultraviolet light (maximum efficiency $72 \%$ at $300 \mathrm{~nm}$ ). The resolution of the monochromator is $1 \mathrm{~nm}$. For the detection of signals, a photomultiplier tube (PMT Hamamatsu R636-10) connected to another Lock in Amplifier is used. With this configuration, it is possible to record SHG and THG signals in distinct sets of measurements by tuning the monochromator in different spectral regions. By detecting SHG or THG images in the forward direction and TPEF images in the backward direction, our experimental apparatus allows the collection of two non-linear optical signals simultaneously.

For the neurodegeneration studies, modifications have been introduced in order to increase the sensitivity of the experimental set-up. A $340 \mathrm{~nm}$ interference filter (Hoya U 340) is used, instead of the monochromator, for the detection of all the bandwidth of the THG signal. Moreover, a more sensitive photomultiplier tube with a preamplifier unit (PMT Hamamatsu H9305-04) is employed.

\subsection{Biological sample}

We have utilized the nematode worm Caenorhabditis elegans ( $C$. elegans) which has emerged as a particularly convenient and versatile model organism, for our analysis. C. elegans is a small ( $1 \mathrm{~mm}$ long and $80 \mu \mathrm{m}$ in diameter) free-living hermaphroditic, nematode worm that completes a life cycle in 2.5 weeks at $25^{\circ} \mathrm{C}$. Animals can be grown on a diet of Escherichia coli, on agar plates or in liquid medium. The simple body plan and transparent nature of both the egg and the cuticle have facilitated the exceptionally detailed developmental and anatomical characterization of this animal. The broad range of genetic and molecular techniques applicable in C. elegans allows a unique line of investigation into fundamental problems in biology (Silva et al., 2006). 
We followed standard procedures for $C$. elegans strain maintenance, crosses and other genetic manipulations (Brenner, 1974). Nematode rearing temperature was kept at $20^{\circ} \mathrm{C}$. Before each experiment, nematodes at the L1 stage of development were anaesthetized by immersing to $20 \mathrm{mM}$ of sodium azide $\left(\mathrm{NaN}_{3}\right)$, and subsequently mounted on glass slides. The strains used for the neurodegeneration studies were the following: wild-type $\mathrm{N} 2$ Bristol isolate, deg-3(u662)V, mec-4(u231)X, N2; Ex[ $p_{\text {mec-4 GFP] }}$ and mec-4(u231)X;Ex[ $\left.p_{\text {mec }-4} \mathrm{GFP}\right]$.

\section{Results and discussion}

Fig. 2 depicts TPEF signals arising from neurons located in the tail of transgenic worms. In this transgenic line, green fluorescence protein (GFP) is expressed under the control of the mec-4 promoter in the six mechanoreceptor neurons of the animal. Two of these touch receptor neurons are located in the posterior part of the worm, close to the tip of the tail, away from the gut autofluorescence. Two different kinds of GFP markers have been used, diffuse GFP (Fig. 2(a)) and oriented GFP (Fig. 2(b)). The first one is localized in the cytoplasm of the mechanoreceptor neurons of the animal, while the second is attached to the membrane of the neuron. Scanning was performed at a specific $z$ position, where the TPEF signal emanating from the neuron axon was maximal. The dimensions of the scanned region were $12 \mu \mathrm{m} \times 6 \mu \mathrm{m}$. The absorption and emission maxima for GFP are 489 and 508, respectively. Although, the emission wavelength of our laser, for TPEF measurements, is not optimal for the excitation of the GFP molecules (less than $20 \%$ is the absorption efficiency of the GFP at $514 \mathrm{~nm}$ ) it was observed that not only the neuronal cell bodies $(2-3 \mu \mathrm{m})$ but and others anatomical components such as neuronal axons $(\sim 200 \mathrm{~nm})$ are detectable.

Fig. 3 presents the combination of the three different non-linear imaging contrast modalities (TPEF in green, SHG in blue and THG in red). The signals were recorded from the anterior part of $C$. elegans mutants. In these transgenic animals, GFP is expressed under the control of the myo- 2 promoter. The myo- 2 promoter is specific to the pharynx and GFP expression is limited to the cytoplasm of pharyngeal muscle cells. Images were recorder with the lowresolution configuration of the set-up, i.e., by moving the sample with the $x y z$ motorized stages. The dimensions of the scanned

(a)

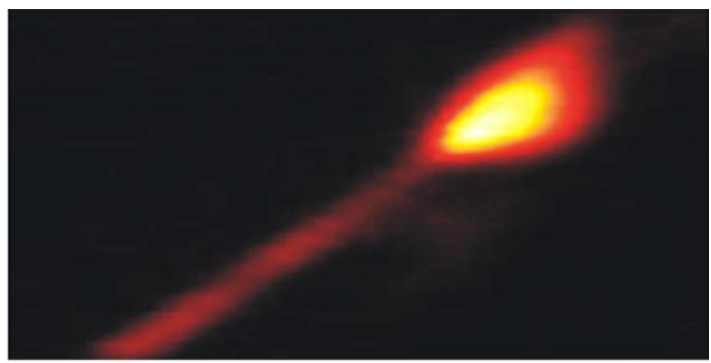

(b)

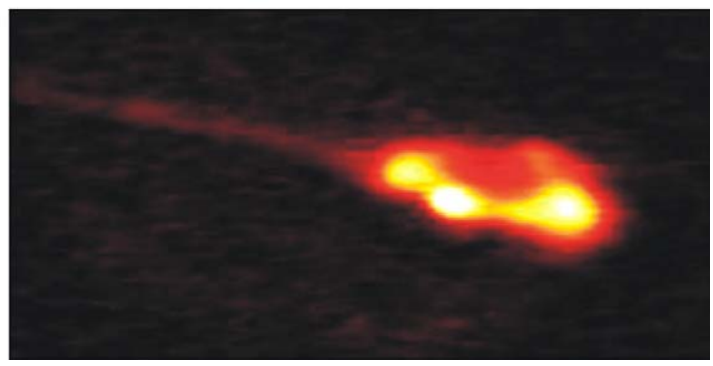

Fig. 2. TPEF images from mechanosensory neurons located in the posterior part of $C$. elegans samples marked with (a) diffused GFP and (b) oriented GFP molecules. region were $60 \mu \mathrm{m} \times 25 \mu \mathrm{m}$ with $1 \mu \mathrm{m}$ resolution. Three different samples were investigated.

The three image modes provide complementary information about the biological sample, as seen in Fig. 3. This is due to the fact that individual, induced signals come from different components. TPEF signals arise from the diffuse GFP molecules expressed in the pharyngeal muscles. By recording these signals it is possible to visualize the inner part of the pharynx.

Highly ordered structures generate a high level of SHG signal. The endogenous structures of well-ordered protein assemblies in the pharyngeal muscles, such as myosin thick filaments, are the main contributors to recorded SHG signals (Plotnikov et al., 2006). GFP molecules, due to their random orientation in the pharynx region do not contribute to the SHG signal. In Fig. 3(a) and (b) the SHG signal arises from the interior of the pharyngeal muscle. In Fig. 3(c), the structure of the longitudinal somatic muscles can be clearly visualized through the SHG measurements.

Structural and functional information can be obtained by recording THG signals, given the sensitivity of these signals to changes of the refractive index of the medium (Squier and Muller, 2001). The contour, the shape and discontinuities in the C. elegans anterior body segment are detected through THG measurements. Furthermore, high THG intensity signals are collected from the linings of the animal pseudocoelomic cavity (Fig. 3(a) and (b)), while no signal arise from the pharyngeal muscle.

Of special interest is the capacity for three-dimensional (3D) reconstruction of a live organism that should facilitate the detailed description of anatomical features of both wild type and genetically modified animals. In contrast to our previous work (Gualda et al., 2008a) here we present new, more detailed and accurate 3D reconstructions of TPEF, SHG and THG images of the nematode $C$. elegans, in both its anterior and posterior body parts. Furthermore, the time for the data acquisition was constrained

(a)

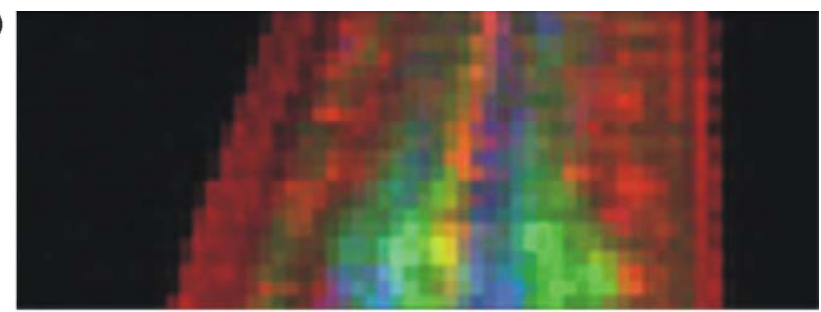

(b)

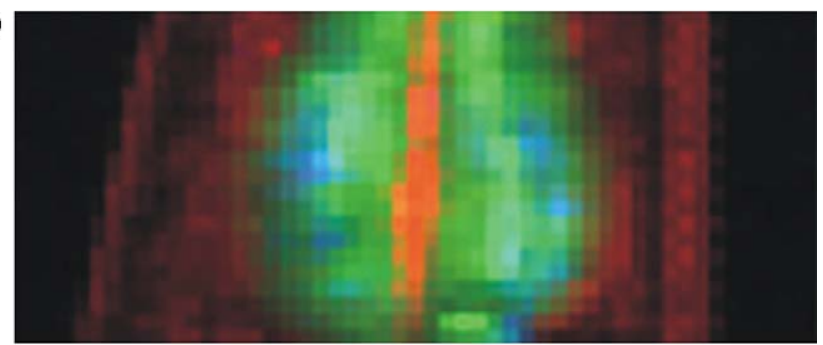

(c)

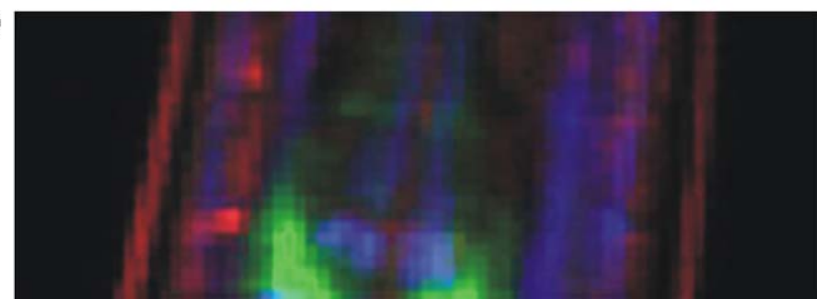

Fig. 3. Non-linear signals (TPEF in green, SHG in blue and THG in red) generated in the pharynx region of worms expressing GFP in the pharyngeal muscles. (For interpretation of the references to colour in this figure legend, the reader is referred to the web version of the article.) 
(a)

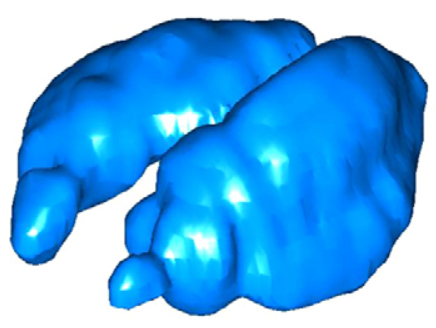

(b)

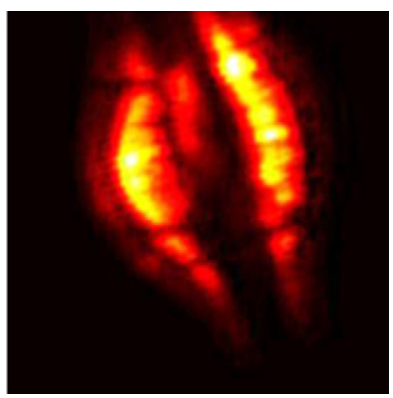

Fig. 4. SHG signal from the anterior bulb of the pharynx of a wild type C. elegans (a) $3 \mathrm{D}$ reconstruction and (b) a slice image.

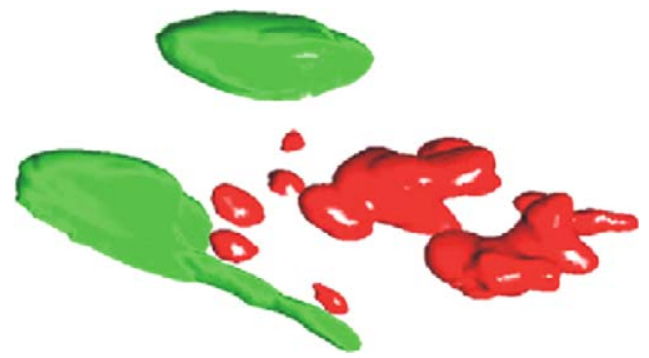

Fig. 5. 3D reconstruction of THG (red) and TPEF (green) images, recorded from the posterior part of a nematode expressing GFP in the mechanoreceptor neurons. (For interpretation of the references to colour in this figure legend, the reader is referred to the web version of the article.)

reducing the side-effects (e.g., thermal, mechanical) to the live biological specimen. Image J (Java-based program, NIH) was used, for processing of the obtained data, for the creation of $3 \mathrm{D}$ reconstruction images.

In Fig. 4(a) the obtained 3D reconstruction of SHG images recorded from the anterior bulb of the pharynx of a wild type $C$. elegans is depicted. The morphology from the muscle of the anterior bulb of the animal can be observed with satisfactory analysis. The main contributors to the recorded signal are the myosin thick filaments (Plotnikov et al., 2006). No fluorescent markers have been used, reducing the photodamage effects. The dimensions of the scanned region were $15 \mu \mathrm{m} \times 15 \mu \mathrm{m}$. Twelve optical sections, $1 \mu \mathrm{m}$ apart, were collected. In Fig. 4(b) one of these sections is shown. We have to note that, the whole procedure of data acquisition for the creation of the 3D reconstruction of SHG images lasted around $25 \mathrm{~min}$. The time needed to record a slice image $(300 \times 300$ points resolution) was $2 \mathrm{~min}$ (due to lock-in detection). The time interval for the realization of the in vivo measurements in $C$. elegans was more than $3 \mathrm{~h}$ during our experiments.
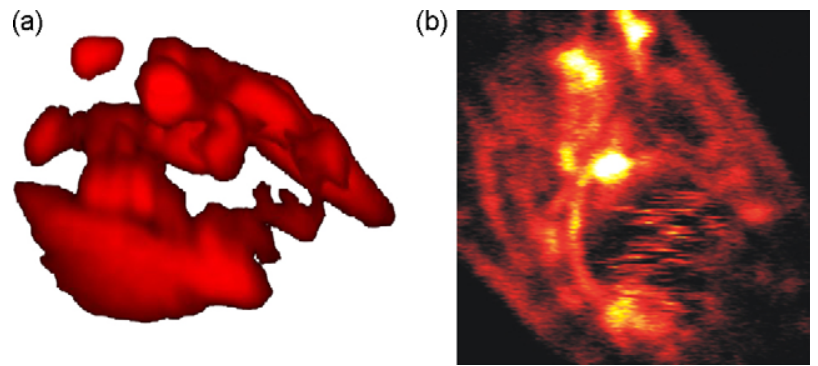

Fig. 6. THG signals from a degenerating neuron located in the posterior part of a $C$ elegans larva: (a) 3D reconstruction and (b) a slice image.
The 3D reconstruction of THG and TPEF images, recorded from the posterior part of the nematode, is shown in Fig. 5. We used strains that express GFP in the cytoplasm of the six mechanoreceptor neurons of $C$. elegans. Two of these neurons are located near the tail of the animal. The precise 3D localization of these two touch receptor neurons and the one neuron axon, expressing diffuse GFP, is easily derived (green surface) from the reconstruction of TPEF images. High THG signals were generated by discontinuities in the region of the tail (red surface). These discontinuities are likely due to lipid depositions (Debarre et al., 2006) or formations of discontinuous refractive index in the tail. The dimensions of the scanned region were $15 \mu \mathrm{m} \times 15 \mu \mathrm{m}$. Twelve optical sections, $1 \mu \mathrm{m}$ apart were obtained.

Moreover, THG imaging modality has been tested as a potential diagnostic tool for providing information related to neurodegeneration phenomena in vivo. Fig. 6(a) illustrates a 3D reconstruction of THG images from a degenerative neuron in the tail of a C. elegans larva. The scanning area was $15 \mu \mathrm{m} \times 15 \mu \mathrm{m}$. Eighteen optical sections, $1 \mu \mathrm{m}$ apart, were recorded. In Fig. 6(b) one of these sections is depicted. The shape of the hole of the degenerative neuron can be clearly observed. The lipids on the membrane of the neuron (Debarre et al., 2006) and discontinuities in the region of the tail are the main sources of the high THG signal. THG signal also arises from the contour of the posterior part of the worm. Additionally, it is worth to mention that, the movement of the nucleus in the cavity of the degenerative neuron of the nematode is detectable via THG measurements (Fig. 6(b) slice image). Thus, THG imaging measurements permit not only the recognition of structural features but also the identification of biological processes occurring in vivo in degenerating neurons. However, it is not feasible the precise monitoring and delineation of the fast movement of the nucleus of the degenerative neuron due to time constrains (the time for acquisition of a high resolution $(300 \times 300$ points) THG image was $2 \mathrm{~min}$ ).

\section{Conclusions}

The in vivo, non-destructive, non-linear imaging microscopy has the potential to offer new insights into the morphological changes and the complex developmental processes in many biological settings. In the present study, consummative and specific information about the anatomy and the morphology of the nematode $C$. elegans were extracted, by implementing a combination of THG, SHG and TPEF image contrast modalities on the same microscope. Additionally, 3D reconstructions of TPEF, SHG and THG images were performed in order to achieve the three dimensional delineation of organs (pharynx) and particular cell types (neurons) of the worm. Furthermore, THG imaging technique has proved to be unique, very useful diagnostic tool providing information related to a specific problem with high biological importance (neuronal degeneration).

\section{Acknowledgements}

This work was supported by the ICT-Collaborative project "FAST-DOT" (Grand Agreement Number 224338) and by the Marie Curie Transfer of Knowledge project "NOLIMBA" (MTKD-CT-2005029194).

\section{References}

Brenner, S., 1974. The genetics of Caenorhabditis elegans. Genetics 77, 71-94.

Campagnola, P.J., Loew, L.M., 2003. Second-harmonic imaging microscopy for visualizing biomolecular arrays in cells, tissues and organisms. Nature Biotechnology 21, 1356-1360.

Debarre, D., Supatto, W., Beaurepaire, E., 2005. Structure sensitivity in thirdharmonic generation microscopy. Optics Letters 30, 2134-2136. 
Debarre, D., Supatto, W., Pena, A.M., Fabre, A., Tordjmann, T., Combettes, L. Schanne-Klein, M.C., Beaurepaire, E., 2006. Imaging lipid bodies in cells and tissues using third-harmonic generation microscopy. Nature Methods 3, 47-53.

Gualda, E.J., Filippidis, G., Voglis, G., Mari, M., Fotakis, C., Tavernarakis, N., 2007. In vivo imaging of anatomical features of the nematode Caenorhabditis elegans using non-linear (TPEF-SHG-THG) microscopy. In: Wilson, T., Periasamy, A (Eds.), Proceedings of the Society of Photo-optical Instrumentation Engineers, Confocal Multiphoton and Nonlinear Microscopic Imaging III 6630. p. 663003.

Gualda, E.J., Filippidis, G., Voglis, G., Mari, M., Fotakis, C., Tavernarakis, N., 2008a. In vivo imaging of cellular structures in Caenorhabditis elegans by combined TPEF, SHG and THG microscopy. Journal of Microscopy 229, 141-150.

Gualda, E.J., Filippidis, G., Mari, M., Voglis, G., Vlachos, M., Fotakis, C., Tavernarakis, N., 2008b. In vivo imaging of neurodegeneration in Caenorhabditis elegans by third harmonic generation microscopy. Journal of Microscopy 232, 270-275.

Mohler, W., Millard, A.C., Campagnola, P.J., 2003. Second harmonic generation imaging of endogenous structural proteins. Methods 29, 97-109.

Plotnikov, S.V., Millard, A.C., Campagnola, P.J., Mohler, W.A., 2006. Characterization of the myosin-based source for second-harmonic generation from muscle sarcomeres. Biophysical Journal 90, 693-703.
Psilodimitrakopoulos, S., Santos, S.I.C.O., Amat-Roldan, I., Thayil, A.K.N., Arigas, D., Loza-Alvarez, P., 2009. In vivo pixel resolution mapping of thick filaments' orientation in nonfibrillar muscle using polarization-sensitive second harmonic generation microscopy. Journal of Biomedical Optics 14, 014001.

Reshak, A.H., Sarafis, V., Heintzmann, R., 2009. Second harmonic imaging of chloroplasts using the two-photon laser scanning microscope. Micron 40, 378-385.

Silva, L.F., Brayner, F.A.S., Santos, A.C.O., Lanfredi, R.M., Peixoto, C.A., 2006. Identification and location of albumin-like antigens in third-stage larva of $W$. bancrofti, in adult forms of Litomosoides chagasfilhoi and in the free-living nematode Caenorhabditis elegans. Micron 37, 666-674.

Squier, J., Muller, M., 2001. High resolution nonlinear microscopy: a review of sources and methods for achieving optimal imaging. Review of Scientific Instruments 72, 2855-2867.

Sun, C.K., Chu, S.W., Chen, S.Y., Tsai, T.H., Liu, T.M., Lin, C.Y., Tsai, H.J., 2004. Higher harmonic generation microscopy for developmental biology. Journal of Structural Biology 147, 19-30.

Zipfel, W.R., Williams, R.M., Webb, W.W., 2003. Nonlinear magic: multiphoton microscopy in the biosciences. Nature Biotechnology 21, 1369-1377. 